DOI: $10.1590 / 1089-6891 v 20 \mathrm{e}-50520$

RECURSOS PESQUEIROS E ENGENHARIA DE PESCA

\title{
IMPLICAÇÕES DE EXTRATO DE Erythrina crista-galli COMO ANSIOLÍTICO PARA Carassius auratus
}

\section{IMPLICATIONS OF Erythrina crista-galli EXTRACT AS ANTISIOLYTIC FOR Carassius auratus}

\author{
Mayara Schueroff Siqueira1'ORCID - http://orcid.org/0000-0003-2309-4743 \\ Rebeca Maria Sousa ${ }^{*}$ ORCID - http://orcid.org/0000-0002-1195-3304 \\ Cleide Adriane Signor Tirloni² ORCID - http://orcid.org/0000-0003-3007-6877 \\ Karime Sater Gebara² ORCID - http://orcid.org/0000-0002-0860-3047 \\ Taline B. Stefanello Catelan² ORCID - http://orcid.org/0000-0003-4335-8881 \\ Claucia Aparecida Honorato² ORCID - http://orcid.org/0000-0002-7161-4996
}

1Universidade Federal de Mato Grosso do Sul, Campo Grande, MS, Brasil.
2Universidade Federal de Grande Dourados, Dourados, MS, Brasil.
${ }^{*}$ Autora para correspondência - $\underline{\text { rebekah.007@hotmail.com }}$

\section{Resumo}

A fitoterapia vem sendo utilizada em criação de peixes a fim de promover estabilidade no ambiente de cultivo e na profilaxia de doenças, contribuindo para a melhoria do bem-estar animal. Dentre os fitoterápicos, Erythrina crista-galli tem efeito sedativo e atividade antioxidante e antimicrobiana, além de funcionar como calmante natural. Na ausência de formulações para uso em peixes cultivados, surgem protocolos terapêuticos repletos de incertezas quanto à sua eficácia, ao impacto ambiental e ao perfil hematológico dos peixes. No ensaio de tolerância aguda da Erythrina crista-galli, foram utilizados exemplares de Carassius auratus, expostos às concentrações de controle (zero), 50, 100 e $200 \mathrm{mgL}^{-1}$, com três repetições, por 96 horas. Foi observada a sobrevivência de $100 \%$ em todos os tratamentos. Estes resultados revelam que o extrato de Erythrina crista-galli não apresenta toxicidade para peixes. O balanço eletrolítico plasmático não apresentou mudanças. A utilização de até 100 $\mathrm{mgL}^{-1}$ não promoveu mudanças na trocas gasosas. $\mathrm{O} \mathrm{pH}$ e a concentração de bicarbonato e glicose foram crescentes até a concentração de $100 \mathrm{mgL}^{-1}$. Conclui-se que a utilização de Erythrina cristagalli para exposição aguda de Carassius auratus não promove mortalidade, mas provoca alterações indesejáveis nos parâmetros fisiológicos sanguíneos quando utilizadas doses acima de $100 \mathrm{mgL}^{-1}$.

Palavras-chave: fitoterápicos, aquários, toxicidade

\begin{abstract}
Phytotherapy has been used in fish breeding to promote stability in the cultivation environment and in the proflatic management reflecting on animal heath. Erythrina crista-galli has a sedative effect and antioxidant and antimicrobial activity, besides functioning as a natural tranquilizer. In the absence of formulations for use in cultivated fish, therapeutic protocols emerge without studies that reveal their characteristics and actions in the animal organism. Therefore, this study evaluated the effects of acute exposure of Carassius auratus to Erythrina crista-galli extract evaluating the physiological blood parameters. In the acute tolerance assay of Erythrina crista-galli, specimens of Carassius auratus were used, exposed to control concentrations (zero), 50, 100 and $200 \mathrm{mg} \mathrm{L}^{-1}$, with three replications, for 96 hours. Survival of $100 \%$ was observed in all treatments. These results reveal that the extract of
\end{abstract}


Erythrina crista-galli does not present toxicity to this species of fish. The plasmatic electrolytic balance showed no changes. The use of up to $100 \mathrm{mg} \mathrm{L}^{-1}$ did not promote changes in gaseous exchanges. The $\mathrm{pH}$ and the concentration of bicarbonate and glucose were increasing until the concentration of 100 $\mathrm{mg} \mathrm{L}^{-1}$. It is concluded that the use of Erythrina crista-galli for acute exposure of Carassius auratus does not promote mortality, but causes undesirable alterations in blood physiological parameters when doses above $100 \mathrm{mg} \mathrm{L}^{-1}$ are used.

Keywords: Herbal medicines. Aquariums. Toxicity

Recebido em: 06 de dezembro de 2017.

Aceito em 06 de novembro de 2018.

\section{Introdução}

Práticas realizadas na aquicultura frequentemente expõem os peixes a intempéries que alteram sua homeostasia ${ }^{(1)}$. Alguns estímulos promovem mudanças comportamentais e fisiológicas que resultam em diminuição do desenvolvimento e em aumento da mortalidade ${ }^{(2)}$. Isso não se diferencia no manejo e na manutenção das espécies de peixes ornamentais. Dentre os peixes de maior popularidade na aquariofilia, encontra-se o gênero Carassius, pela docilidade e pela rusticidade diante das condições de confinamento em aquários ${ }^{(3)}$.

Com a finalidade de diminuir os efeitos do estresse, tem-se utilizado fitoterápicos, que, por sua vez, têm demonstrado eficácia para contornar eventuais transtornos nas práticas de manejo aplicadas no cotidiano da criação de peixes ${ }^{(4)}$. A fitoterapia vem sendo utilizada em criação de peixes a fim de promover estabilidade no ambiente de cultivo e na profilaxia de doenças, contribuindo para a melhoria do bem-estar animal ${ }^{(2)}$.

Em um país biologicamente tão rico, pesquisas com plantas medicinais deveriam ser mais incentivadas, visto que há grande tendência mundial de aumento na utilização de fitoterápicos ${ }^{(5,6)}$. Esses produtos apresentam menor número de efeitos colaterais que os medicamentos sintéticos, o que nem sempre é confirmado pelas pesquisas ${ }^{(7)}$. Algumas plantas apresentam a capacidade de atuar no comportamento, no humor, no pensamento e nas sensações, e o entendimento de seus mecanismos de ação, segurança e eficácia constitui um desafio para os pesquisadores ${ }^{(8,9)}$. Dentre as plantas medicinais com efeitos no sistema nervoso central, destacam-se as do gênero Erythrina, as quais são utilizadas como tranquilizantes ${ }^{(9)}$.

A Erythrina crista-galli é conhecida no Brasil como "Corticeira". Em estudos farmacológicos, vem demonstrado atividades antinociceptiva, ansiolítica/sedativa, relaxante muscular e antibacteriana de extratos de Erythrina velutina ${ }^{(10,11)}$. No entanto, estudos toxicológicos que comprovem a segurança de seu uso, apesar de muito importantes ${ }^{(9)}$, ainda são muito escassos.

Portanto, este trabalho avaliou os efeitos da exposição aguda do Carassius auratus ao extrato de Erythrina crista-galli avaliando os parâmetros fisiológicos sanguíneos. 


\section{Material e métodos}

As folhas de Erythrina crista-galli foram coletadas no município de Dourados, estado de Mato Grosso do Sul, Brasil, durante a estação de estiagem. A identidade botânica da planta foi confirmada através da comparação a um espécime que se encontra depositado no Herbário da Universidade Federal da Grande Dourados/UFGD, sob o registro 4879.

A secagem das folhas (Figura 1) foi realizada ao ar à temperatura ambiente, trituradas em moinho de faca e submetidas à extração por maceração com metanol, exaustivamente e posteriormente filtradas e concentradas em rotaevaporador sob pressão reduzida, para a obtenção do extrato metanólico. $\mathrm{O}$ infuso assim obtido foi filtrado sob vácuo, liofilizado (Freezone 4.5, Labconco) e estocado a $5{ }^{\circ} \mathrm{C}$ para uso posterior.

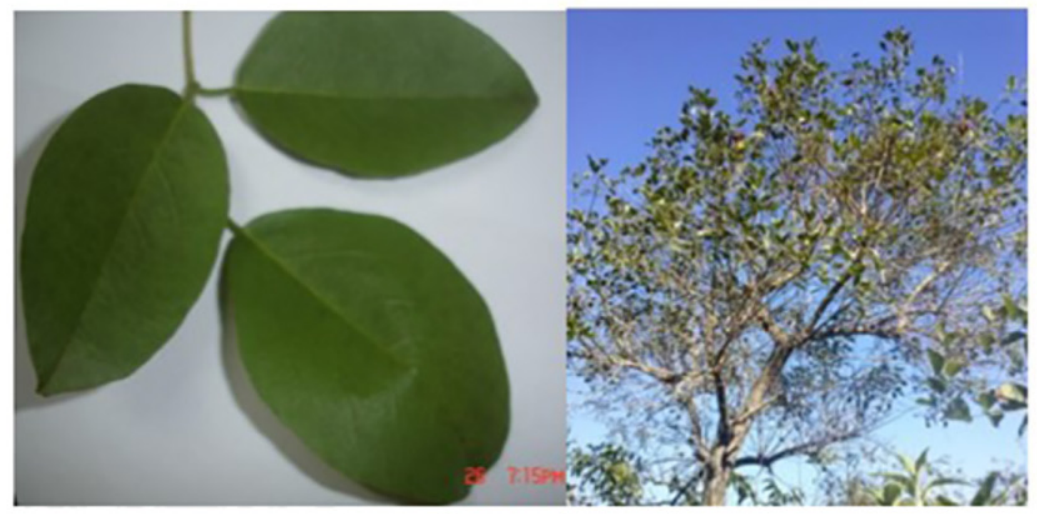

Figura 1. Folhas e árvore de Erythrina crista-galli.

Foram utilizados exemplares de Carassius auratus, com peso de 5,53 $\pm 1,04 \mathrm{~g}$, aclimatados ao ambiente durante 15 dias. Nesse período, os peixes foram mantidos em caixa de $500 \mathrm{~L}$, com aeração constante, foram alimentados até a saciedade, duas vezes ao dia, com ração comercial extrusada (32\% $\mathrm{PB})$.

No ensaio de tolerância aguda da Erythrina crista-galli, os peixes foram submetidos a quatro tratamentos compostos por concentrações de controle (zero), 50, 100 e $200 \mathrm{mgL}^{-1}$, com três repetições. Os testes foram conduzidos em unidades experimentais de $20 \mathrm{~L}$ de água, contendo cinco peixes, com total de 15 peixes por concentração, em sistema estático, com sifonagem e posterior exposição da água contendo as respectivas concentrações dos extratos. O período de exposição dos peixes foi de 96 horas, com fotoperíodo de 12 horas de luz.

Para a realização dos testes de toxicidade aguda, a alimentação foi suspensa 24 horas antes do início do experimento, e a relação massa-volume foi mantida próximo de $1 \mathrm{~g} / \mathrm{L}$, em conformidade com as recomendações das normas de ensaio de toxicidade aguda com peixes, contidas na Associação Brasileira de Normas Técnicas - ABNT NBR 15088:2011 ${ }^{(12)}$.

Os dejetos foram sifonados e a água reposta com a mesma concentração. Diariamente, foram mensurados a temperatura da água $\left({ }^{\circ} \mathrm{C}\right)$, o oxigênio dissolvido $\left(\mathrm{mgL}^{-1}\right)$ e o $\mathrm{pH}$. As variáveis liminológicas mensuradas mantiveram-se estáveis durante o período experimental. $\mathrm{O}$ ensaio foi aprovado pelo Comitê de Ética em Pesquisa com Animais do Centro Universitário da Grande Dourados/CEUA, protocolo: 003/14. 
A tolerância dos peixes foi aferida pela sobrevivência às concentrações testadas a cada hora durante as seis primeiras horas do ensaio, posteriormente com intervalos de seis horas até 24 horas de exposição, e diariamente até 96h. Após o período de exposição à Erythrina crista-galli, todos os peixes de cada aquário foram rapidamente contados e capturados para coleta de sangue, com seringa de três $\mathrm{mL}$ heparinizada pela veia caudal, e coleta das brânquias. O sangue foi analisado em aparelho de hemogasometria (Cobas HB121 - Roche Diagnóstica Brasil, São Paulo, SP, Brasil). Foram mensurados os íons Sódio $\left(\mathrm{Na}^{+}\right)$, Potássio $\left(\mathrm{K}^{+}\right)$, Cloreto $\left(\mathrm{Cl}^{+}\right)$e Cálcio $\left(\mathrm{Ca}^{2+}\right)$. Foram também determinados parâmetros respiratórios no sangue de $\mathrm{pH}, \mathrm{H}^{+}$, concentração de bicarbonato $\left(\mathrm{HCO}_{-3}\right)$, pressão parcial de oxigênio $\left(\mathrm{PaO}_{2}\right)$ e pressão parcial de dióxido de carbono $\left(\mathrm{PaCO}_{2}\right)$.

O delineamento foi inteiramente casualizado (DIC), com quatro concentrações e três repetições. As análises de variância (ANOVA) e as médias foram comparadas pelo teste de Tukey $(\mathrm{p}<0,05)$.

\section{Resultados e discussão}

Os índices médios para oxigênio dissolvido foram de 5,70 $\pm 0,2 \mathrm{mgL}^{-1}$, para temperatura média de $26,27 \pm 1,20^{\circ} \mathrm{C}$, de $\mathrm{pH}$ de 7,95 $\pm 0,03$. As pequenas variações encontradas nos valores de $\mathrm{pH} \mathrm{e}$ oxigênio dissolvido da água ocorreram dentro de amplitudes consideradas ótimas para a criação da maioria das espécies de peixes ${ }^{(13)}$. Observou-se sobrevivência de $100 \%$ em todos os tratamentos no período de estudo (96h).

As concentrações gasosas $\left(\mathrm{PaO}_{2}\right.$ e $\left.\mathrm{PaCO}_{2}\right)$ não apresentaram mudanças até a utilização de $100 \mathrm{mg} \mathrm{L}^{-1}$ de Erythrina crista-galli. A utilização de $200 \mathrm{mg} \mathrm{L}^{-1}$ de Erythrina crista-galli promoveu aumento na $\mathrm{PaO}_{2}$ e na $\mathrm{PaCO}_{2}$. A utilização de fitoterápicos dissolvidos na água pode causar reações adversas secundárias, tais como acidose e estresse osmótico, devido à parada respiratória e à insuficiente troca gasosa e iônica entre o sangue e a água ${ }^{(14)}$. Segundo Keen \& Gamperl ${ }^{(15)}$, as variações $\mathrm{PaO}_{2}$ e $\mathrm{PaCO}_{2}$ podem ser compensadas por alterações cardíacas, promovendo hiperventilação das brânquias.

Não foram observadas diferenças nas concentrações de sódio e cálcio e hidrogênio plasmático (Tabela 1). A concentração de potássio sanguíneo dos peixes em exposição aguda apresentou diminuição em relação ao controle, quadro típico de hipotassemia. O sangue de peixes tem salinidade próxima a $9 \%$, sendo que $77 \%$ dos sais no sangue são sódio $\left(\mathrm{Na}^{+}\right)$e cloreto $\left(\mathrm{Cl}^{+}\right)$, o que permite a regulação com o ambiente aquático. Aumento da osmolaridade no plasma $\left(\mathrm{Na}^{+}, \mathrm{Cl}^{+}, \mathrm{K}^{+} \mathrm{e} \mathrm{Mg}^{+}\right)$foi reportado para a truta arco-íris (Oncorhynchus mykiss), submetida à salinidade por Al-Jandal e Wilson ${ }^{(16)}$. A diminuição dos níveis séricos de potássio está associada principalmente à perda de água para o meio externo $^{(17)}$ ou às mudanças de $\mathrm{pH}$ sanguíneos ${ }^{(18)}$.

As concentrações de sódio, cloreto e cálcio plasmático mantiveram-se constantes (Tabela 1), o que indica que os procedimentos experimentais aparentemente não tiveram intensidade nem duração suficientes para induzir alterações no balanço eletrolítico nesta espécie de peixe. O equilíbrio de sais no plasma pode ser alterado em resposta às mudanças da quantidade de água no sangue que passa pelas brânquias, alterando, também, as trocas de íons entre os meios interno e externo ${ }^{(19)}$. $\mathrm{O}$ estresse de manejo provoca alterações nos níveis de sódio, cloreto, cálcio e potássio de truta arco-íris Oncorhynchus mykiss ${ }^{(20)}$.

Alterações nesse balanço de íons podem ser decorrentes dos efeitos de poluentes nos órgãos diretamente 
envolvidos na osmorregulação, no sistema endócrino, no metabolismo ou nos processos de transporte ativo. Assim, os níveis iônicos no sangue, determinados pela osmolaridade ou por concentrações iônicas específicas, são biomarcadores sensíveis de exposição de peixes a agentes químicos e seus efeitos $^{(21)}$

Tabela 1. Parâmetros sanguíneos de Carassius auratus após exposição aguda a Eritrina cristagalli

\begin{tabular}{lcccc}
$\begin{array}{l}\text { Parâmetros } \\
\text { respiratórios }\end{array}$ & Controle & $\mathbf{5 0 ~} \mathbf{~ m g ~ L}$ & $\mathbf{1 0 0} \mathbf{~ m g ~ L}^{-\mathbf{1}}$ & $\mathbf{2 0 0} \mathbf{~ m g ~ L}^{-\mathbf{1}}$ \\
& & & & \\
\hline $\mathrm{PaO}_{2}$ & $63,55 \pm 10,54 \mathrm{~b}$ & $69,20 \pm 3,1 \mathrm{~b}$ & $41,80 \pm 4,24 \mathrm{c}$ & $80,05 \pm 4,31 \mathrm{a}$ \\
$\mathrm{PaCO}_{2}$ & $7,25 \pm 0,07 \mathrm{~b}$ & $8,20 \pm 0,16 \mathrm{~b}$ & $8,45 \pm 0,07 \mathrm{~b}$ & $10,85 \pm 1,06 \mathrm{a}$ \\
\hline Eletrólitos & & & & \\
\hline $\mathrm{Na}^{+}$ & $151,05 \pm 11,10$ & $148,50 \pm 5,30$ & $149,70 \pm 5,80$ & $150,85 \pm 7,57$ \\
$\mathrm{~K}^{+}$ & $7,20 \pm 1,55^{\mathrm{a}}$ & $3,29 \pm 1,12 \mathrm{c}$ & $5,60 \pm 1,83 \mathrm{~b}$ & $4,88 \pm 1,89 \mathrm{~b}$ \\
$\mathrm{iCa}^{+}$ & $0,17 \pm 0,01$ & $0,19 \pm 0,02$ & $0,22 \pm 0,01$ & $0,20 \pm 0,01$ \\
$\mathrm{H}^{+}$ & $43,00 \pm 11,31$ & $37,30 \pm 6,62$ & $42,95 \pm 16,62$ & $40,45 \pm 13,93$ \\
\hline
\end{tabular}

$\mathrm{PaO}_{2}$ Pressão de oxigênio, $\mathrm{PaCO}_{2}$ Pressão de dióxido de carbono, $\mathrm{Na}$ - sódio, $\mathrm{K}$ - potássio, $\mathrm{ICa}-$ íon cálcio, $\mathrm{H}+$ - eletrólitos. Letras distintas reportam diferença estatistica pelo teste de Tukey $(\mathrm{P}>0,05)$.

A

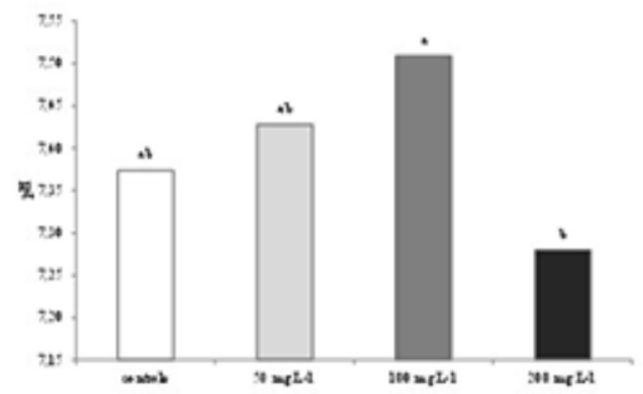

B

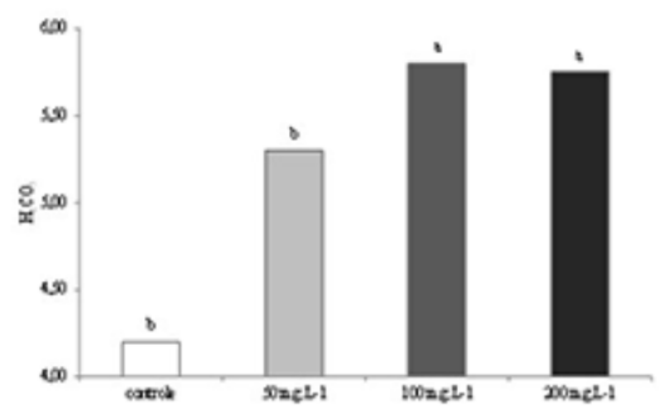

$\mathrm{C}$

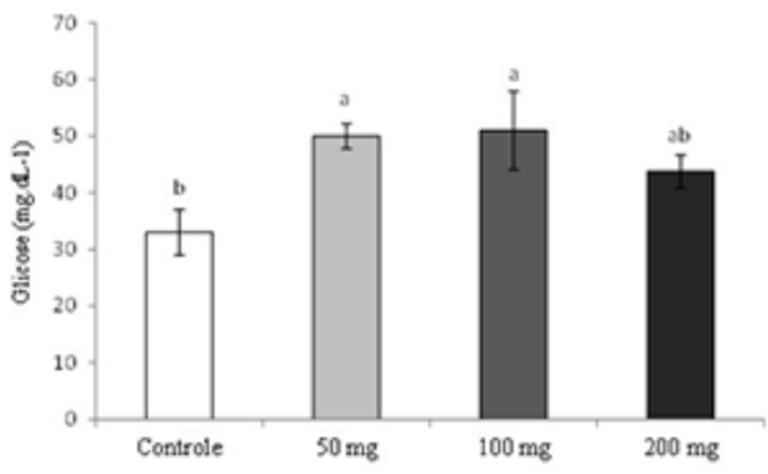

Figura 2. (a) $\mathrm{pH}$ e (b) $\mathrm{H}_{2} \mathrm{CO}_{3}$ (c) glicose sanguíneos de Carassius auratus após exposição aguda a Erythrina crista-galli. Letras distintas reportam diferença estatística pelo teste de Tukey $(\mathrm{P}>0,05)$. 
$\mathrm{O}$ pH sanguíneo aumentou até a concentração de $100 \mathrm{mgL}^{-1}$ (Figura 2A). A utilização de concentrações crescentes de Erythrina crista-galli induziu ao aumento de bicarbonato plasmático (Figura 2B). O estudo da variável bicarbonato plasmático $\left(\mathrm{HCO}_{3}^{-}\right)$permite avaliar a resposta do organismo diante de variações do $\mathrm{pH}$ no sangue. Segundo Luna ${ }^{(2)}$, o bicarbonato é responsável por mais de $50 \%$ da capacidade-tampão extracelular. A concentração de bicarbonato plasmático está diretamente relacionada à elevação na $\mathrm{PaCO}_{2}$ (Tabela 1), pois o mecanismo compensatório eleva as taxas de bicarbonato a fim de neutralizar o excesso de $\mathrm{CO}_{2}$ presente no sangue ${ }^{(23)}$.

O nível de glicose plasmática apresentou aumento em relação ao grupo controle. A glicose é um dos indicadores mais utilizados na avaliação do estresse em peixes como resposta secundária. Sua elevação deve-se à presença de algum fator estressante para suprir a maior demanda energética, característica de situações desfavoráveis ${ }^{(1)}$.

Segundo Zahl, Samuelsen \& Kiessling ${ }^{(14)}$, o uso de alguns fitoterápicos pode ter efeitos secundários indesejados que reduzem o bem-estar dos peixes, promovendo alterações na homeostasia. Segundo Inoue et al. ${ }^{(24)}$, a transferência dos peixes de local provoca estímulos adversos suficientes para iniciar as respostas metabólicas ao estresse, resultando em aumento da glicose do plasma. Inoue et al. ${ }^{(24)}$ afirmam que os aumentos são interpretados de forma que o cérebro detecte um ou mais estímulos adversos. Dois eixos metabólicos são ativados: CPI (cérebro-pituitária-inter-renais) e CSC (cérebro, células simpáticas de cromafins). No entanto, neste trabalho, o grupo controle que também passou pelo mesmo manejo não apresentou elevação da glicose plasmática, podendo assim ser atribuída essa resposta à presença de Erythrina crista-galli.

\section{Conclusão}

A utilização de Erythrina crista-galli para exposição aguda de Carassius auratus não acarreta letalidade, mas promove alterações indesejáveis nos parâmetros fisiológicos sanguíneos. Portanto, sua ação ansiolítica não traz benefícios para o manejo desta espécie de peixe.

\section{Agradecimentos}

Apoio financeiro FUNDECT - 0143/12

\section{Referências}

1. Diniz NM, Honorato CA. Algumas alternativas para diminuir os efeitos do estresse em peixes de cultivo revisão. Arquivo de Ciências Veterinária e Zoologia UNIPAR. 2012; 15(2): p. 149-154.

2. Abe HA, Dias JAR, Reis RGA, Couto MVS, Meneses JO, Fujimoto RY. Extrato aquoso de canela como promotor de crescimento para larvas do peixe ornamental amazônico Pyrrhulina brevis. Bol. Ind. Anim.. 2016; 73(4): p. 267-271.

3. Taghizadeh V. Effects of acidic water in combination with aliminum on swimming behavior and survival of yolk-sac larval in Goldifish (Carassius auratus gibelio). Springer Plus. 2013; 2(1): p. 1-6. 
4. Gholipourkanani H, Ahadizadeh S. Use of Propofol as an anaesthetic and its efficacy on some hematological values of ornamental fish Carassius auratus. Springer Plus. 2013; 2(76).

5. Carvalho ACB, Balbino EE, Maciel A, Perfeito JPS. Situação do registro de medicamentos fitoterápicos no Brasil. Rev Bras de Farmacogn. 2008; 18: p. 314-319.

6. Veiga-Junior VF. Estudo do consumo de plantas medicinais na Região Centro-Norte do Estado do Rio de Janeiro: aceitação pelos profissionais de saúde e modo de uso pela população. Rev Bras Farmacong. 2008;(18): p. 308-313.

7. Calixto JB. Efficacy, safety, quality control, marketing and regulatory guidelines for herbal medicines (phytoterapeutic agents). Bras J Med Biol Res. 2000;(33): p. 179-189.

8. Carlini EA, Rodrigues E, Mendes FR, Tabach R, Gianfratti B. Treatment of drug dependence with Brazilian herbal medicines. Rev. Bras. Farmacogn. 2006; 16: p. 690-695.

9. Craveiro CS, Carvalho DMM, Nunes RdS, Fakhouri R, Rodrigues SA, Teixeira-Silva F. Toxicidade aguda do extrato aquoso de folhas de Erythrina velutina em animais experimentais. Revista Brasileira de Farmacognosia. 2008 Dezembro;(18): p. 739-743.

10. Virtuoso S, Davet A, Dias JFG, Cunico MM, Miguel MD, Oliveira AB, et al. Estudo preliminar da atividade antibacteriana das cascas de Erythina velutina Willd., Fabaceae (Leguminoseae). Res Bras Farmacong. 2005; 15: p. 137-142.

11. Santos MRV, Alves PB, Antoniolli AR, Marchioro M. Relaxant effect of the aqueous extract of Erythrina vellutina leaves on rat vas deferens. Res Bras Farmacong. 2007; 17: p. 343-348.

12. ABNT. Associação Brasileira de Normas Técnicas - ABNT NBR 15088:2011. 2011.

13. Urbinati EC, Gonçalves FD. Espécies nativas para piscicultura no Brasil Santa Maria: UFSM; 2005.

14. Zahl I, Samuelsen O, Kiessling A. Anaesthesia of farmed fish: implications for welfare. Fish Physiologyand Biochemistry. 2012; 38(1): p. 201-21.

15. Keen AN, Gramperl AK. Blood oxigenation and cardiorespiratory function in steelhead trout (Oncorhynchus mykiss) challenged with an cute temperature increase and zatebradine-induced bradycardia. Journal of Thermal Biology. 2012; 37(3): p. 201-210.

16. Al-Jandal NJ, Wilson RW. A comparison of osmoregulatory responses in plasma and tissues of rainbow trout (Oncorhynchus mykiss) following acute salinity challenges. Comparative Biochemistry Physiology. 2011; 2: p. 175-181.

17. Polzin DJ. Diagnostic \& staging kidney disease in dogs and cats [Diagnosting \& staging kidney in dogs and cats].; 2008 [cited 2018 agosto 20. Available from: www.chicagovma.org/wp-content/uploads/2014/06/ Nephrology-2008.pdf.

18. Waki F, Martonnelli R, Mosko E, Kogika M. [Classificação em estágios da doença renal crônica em cães e gatos: abordagem clínica, laboratorial e terapêutica ].; 2010 [cited 2018 Outubro 31. Available from: www. scielo.br/scielo.php?script=sci arttest\&pid=s010384782010001000029\&Ing=en\&nrm-iso .

19. McDonald G, Milligan L. Ionic, osmotic and acid-base regualtion in stres Cambrigde; 1997.

20. Tahmasebi-Kohyani A. Effects of dietary nucleotides suplementation on rainbow trout (Oncorhynchus mykiss) performance and acute stress response. Fish Physiology and Biochemistry. 2012; 38(2): p. 431-440.

21. Winkaler U, Silva AdG, Galindo C, Martinez BdR. Biomarcadores histológicos e fisiológicos para o 
monitoramento da saúde de peixes de ribeirões de Londrina, Estado do Paraná. Acta Scientiarum. 2001: p. 507-514.

22. Luna SPL. Equilíbrio ácido-base. In. In Fantoni DT, Cortopassi SRG. Anestesia de cães e gatos. São Paulo: Rocca; 2002. p. 120-129.

23. Souza AP, et al.. Efeitos cardiorespiratórios da buprenorfina em cães anestesiados pelo desfluoreno. Ciência Rural. 2005; 35(6).

24. Inoue AK, et al.. Avaliação de respostas metabólicas do tambaqui exposto ao eugenol em banhos anéstesicos. Acta Amazônica. 2011; 41(2): p. 327-332. 